\title{
Coma Stimulation
}

Sara Bos, MS, BSN

Volume 4

February 26, 1997

Document Number 1

Indexing terms: coma, stimulation, head injury, recovery, rehabilitation

\section{Abstract}

(1) Coma stimulation is a treatment in which a health care professional or a patient's family member systematically applies stimulation to one or more of the patient's five senses, for the purpose of increasing patient responsiveness. The rationale is that exposure to frequent and various sensory stimulation will facilitate both dendritic growth and improve synaptic connectivity in those with damaged nervous systems (Ansell, 1991 [1]; Kater, 1989 [7]). This review was conducted to determine whether research supports the incorporation of a coma stimulation program into routine nursing care. Included studies were of stimulation programs in inpatient settings, analyzing both multimodal and unimodal sensory stimulation. Implications for nursing practice are given, including specific prescriptives for implementing a coma stimulation program and areas for further research.

\section{Statement of the Practice Problem}

(2) Nurses routinely manage the care of comatose, head injured patients on their intensive care and neurology units. Although it is common practice for nurses to talk to a comatose person and to compassionately touch them, current nursing practice does not routinely incorporate structured coma stimulation for comatose, head injured patients. Coma stimulation is a treatment in which a health care professional or a patient's family member systematically applies stimulation to one or more of the patient's five senses, for the purpose of increasing patient responsiveness.

(3) A primary goal of professionals who advocate coma stimulation as a treatment is to increase a patient's responsiveness and, thus, foster recovery (Jones, Hux, Morton-Anderson, \& Knepper, 1994 [6]). The rationale is that exposure to frequent and various sensory stimulation will facilitate both dendritic growth and improve synaptic connectivity in those with damaged nervous systems (Ansell, 1991 [1]; Kater, 1989 [7]). It is possible, then, that coma stimulation will improve both cognitive functioning and environmental interaction of head injured patients. This paper will summarize and evaluate the recent literature relevant to the practice of coma stimulation and consider whether research supports the incorporation of a coma stimulation program into routine nursing care.

\section{Summary of Research}

(4) In recent years, a considerable reduction in the rate of mortality immediately following severe head injury has been achieved by prompt diagnosis, resuscitation, and effective clinical management (Mitchell, Bradley, Welch, \& Britton, 1990 [9]). Coma stimulation is one avenue toward effective clinical management that is addressed in the literature.

\section{Subjects and setting}

(5) All of the studies included in this analysis were conducted in inpatient settings: either acute hospital settings or post-acute, rehabilitation settings. Though subjects were of both genders and covered many age groups, the majority of subjects were men under the age of 30 . Level of coma was described in a variety of ways, though most studies used either the Ranchos Los Amigos (range level II-III) (Jones et al., 1994 [6]; Rader, Alston, \& Ellis, 1989 [11]) and/or the Glasgow Coma Scale (range level 3-8) (Kater, 1989 [7]; Mitchell et al., 1990 [9]; Pierce et al., 1990 [10]; Sisson, 1990 [12]). One case study used diagnosis of a vegetative state as the definition of coma (Wilson, Powell, Elliott, \& Thwaites, 1991 [14]) .

(6) In the vegetative state, as defined by Jennett and Plum (1972 [5]), the patient may have periods of wakefulness when their eyes are open and they move. However, patient responsiveness is limited to postural and reflex movements, and they never speak (Jennett \& Plum, 1972 [5]). Given this definition, the lines differentiating coma and a vegetative state are somewhat blurry, though the latter term is usually reserved for patients who fail to progress in responsiveness. In the article by Wilson et al. (1991 [14]) anecdotal descriptions of patients in the vegetative state were similar to the descriptions of patients who, in other articles, were defined at the low end of the Glasgow Coma Scale. For this reason, the study by Wilson et al. (1991 [14]) was included for review as well. There was no apparent correspondence between location of injury and those who had better outcomes. Subjects included for study had injuries ranging from focal to diffuse injuries.

\section{Type of stimulation}

(7) The studies were divided into two groups: those that used multimodal stimulation (stimulation of all five senses) and those that used unimodal stimulation (stimulation of one sense). Brief examples of what is meant by multimodal stimuli may benefit the reader both in understanding and practical application. For instance, the tactile sense may have been stimulated using a feather, sandpaper, or fur piece, while vision was stimulated using such things as a snow globe or blinking flashlight to encourage 
tracking. The gustatory sense was stimulated with both taste and texture/temperature: swabs of spices on the tongue or popsicles, for instance. Examples of auditory stimulation are given in the unimodal section.

\section{Multimodal}

(8) Several studies used multimodal stimulation as the stimulation intervention. In a comparison study done by Mitchell et al. (1990 [9]), subjects received stimulation to all five senses via stimulus items from a coma kit for one to two times daily. Each session took approximately one hour, during which each of the five senses was stimulated five times. Family members of the patient were instructed in the procedure and provided the stimulation throughout the duration of the patient's coma. (Coma was deemed to have ended when the patient was observed to obey commands and to make purposeful movements.) Subjects in the experimental group were found to have a significant decrease in the mean coma length as well as a faster increase in Glasgow Coma Scale mean weekly scores (Mitchell et al., 1990 [9]).

(9) A study by Kater (1989 [7]) also had positive results. Using an experimental group $(n=15)$ matched with a control group, Kater used a multimodal stimulation intervention strategy. Family members and staff were taught how to perform the interventions as well and were encouraged to apply sensory stimulation whenever they approached the patient. In addition to the informal sessions conducted by family members and staff, formal stimulation sessions were conducted in the experimental group by the researcher twice a day for 45 minutes, six days a week for a 1-3 month period. Kater found that the experimental group had significantly higher cognitive level scores at a three month measure than did those patients in the control group. (Cognitive level scores were determined using the Level of Cognitive Functioning Record; Kater, 1989 [7]).

(10) Kater (1989 [7]) provided some additional insight not seen in other research studies: division of coma stimulation effect based on coma severity. The experimental group was divided into three levels: deep coma (Glasgow coma score 3-6), moderate coma (GCS 7-10), and light coma (GCS 11-14). Kater noted that the difference in cognitive level scores was greatest for those patients in the moderate coma severity group. Patients in deep coma also seemed to benefit from the stimulation, though patients in the light coma severity group demonstrated very little difference from their control group counterparts in their mean cognitive scores (Kater, 1989 [7]).

(11) Wilson et al. (1991 [14]) performed a study of multimodal stimulation in four patients. This stimulation intervention did not stimulate the taste sense because the researcher noted hypersensitive mouths in some patients. After 11 daily ten-minute stimulation interventions by the researcher, each of the four patients showed an increase in one or more of five behavioral measures (including vocalization, eye opening, and extremity movement) (Wilson et al., 1991 [14]).

(12) In studying multimodal sensory stimulation, Rader et al. (1989 [11]) analyzed two additional dimensions of stimulation sessions: interpersonal contact and position. These researchers found in a pilot study that the best changes in general responsiveness level among coma patients receiving multimodal stimulation occurred in the supine, high interpersonal contact condition. In upright positions, increases were moderate (with both low and high interpersonal contact), while a general responsiveness decline was found using the supine, low interpersonal contact condition. Rader et al. used this information to develop the standardized procedure of sensory stimulation using the supine, high interpersonal contact condition. After two stimulation interventions (50 minutes a day for two days) under these conditions, subjects failed to show significant improvement in their mean general responsiveness level (this included eye opening, motor, and vocalization) at three months post intervention (Rader et al., 1989 [11]).

(13) Pierce et al. (1990 [10]) conducted a more vigorous stimulation program than the researchers cited above. Multimodal stimulation sessions lasted from three to eight hours per day and were performed by family members and friends of the patient. The sessions lasted anywhere from 2-32 weeks. The two outcomes measured were patient's Glasgow Outcome Scale Score 10-12 months post-injury and time taken to obey a simple command on two consecutive occasions 24 hours apart. Despite the level of stimulation, the 31 subjects in this study did not emerge from coma faster than those in a control group. In addition, though the patients in the stimulation group had better recovery outcomes more often than those in the control group (a difference of 11\%), it did not reach statistical significance (Pierce et al., 1990 [10]).

\section{Unimodal}

(14) Of the studies that used unimodal stimulation, the auditory sense was most often chosen as the stimulation intervention. In one study by Wilson et al. (1991 [14]), the unimodal stimulation was alternated between the five senses for 11 days, and no significant increase in five defined behavioral measures was found (Wilson et al., 1991 [14]).

(15) In studies using auditory stimulation, Jones et al. (1994 [6]) used one subject to compare four different types of tape recorded auditory stimulation: voices of family and friends, classical music, popular music, and nature sounds. Auditory stimulation was performed with this subject twice a day for a total of 14 days. Though each session lasted approximately 20 minutes, the auditory stimulus accounted for about five minutes of this time. The remaining time was used in taking baseline measurements and allowing for periods of silence. These researchers found an increase in the patient's arousal (defined as increases in pulse, respirations, body and facial movements) following the recordings of the voices of family and friends and no change, or a decrease from baseline status, following the remaining three auditory stimuli (Jones et al., 1994 [6]).

(16) Sisson (1990 [12]) did a similar pilot study of five comatose individuals using auditory stimulation of two currently popular songs on cassette recordings. The results from this researcher were mixed. Of the five subjects studied, three had no measurable response on EEG measurement but did have measurable behavioral response (motor activity or eye opening). The two subjects that did show an alerting response on EEG monitoring had no measurable behavioral response (Sisson, $1990[12]$ ). 


\section{Theoretical Frameworks}

(17) Many of the studies reviewed here did not list a theoretical framework for study or cited parts of two or three theories as support for the study. The theoretical framework most frequently cited was that of sensory deprivation (Jones et al., 1994 [6]; Mitchell et al., 1990 [9]; Rader et al., 1989 [11]). To briefly summarize, sensory deprivation theory holds that patients in a coma experience sensory deprivation because their ability to respond to internal and external stimuli is altered (Davis \& White, 1995 [4]). Because of this alteration, the threshold of activation of the reticular activating system may increase. The practical implication of this theory is that controlled stimulation may meet the higher threshold of the reticular neurons and increase cortical activity (Sosnowski \& Ustik, 1994 [13]).

(18) Another theory of coma stimulation that was only minimally discussed in this literature (Jones et al., 1994[6]; Kater, 1989 [7]) is that of neural plasticity and sensitive windows of development. Neural plasticity has been studied mainly in animals and describes the ability of the mature nervous system to continually undergo changes (Davis \& White, 1995 [4]), in particular, increased dendritic branching and increased numbers of dendritic spines (Ansell, 1991 [1]). These changes suggest growth of synapses and synaptic connectivity (Ansell, 1991 [1]). Because damage to the nervous system (as with traumatic brain injury) may serve as a catalyst for this increase in synaptogenesis (Cotman \& Nieto-Sampedro, 1982 [3]), it is possible that the delivery of stimulation during the time when neural regrowth is occurring may maximize the effects of plasticity. That is, the most benefit from stimulation programs may occur when the patient is closest to his time of injury. An evaluation of the literature to look at subjects' time elapsed since injury may, therefore, help researchers and practitioners draw inferences related to critical windows of opportunity.

\section{Time elapsed since injury}

(19) There was a wide range of post-injury time frames for stimulation intervention both between studies and within individual studies. The subjects in the study by Mitchell et al. (1990 [9]) had the earliest stimulation intervention, being 4-12 days post-injury. The stimulation interventions for other studies were commenced at 42 days post-injury (Jones et al., 1994 [6]), 2-33 months, mean 12.4 months post-injury (Rader et al., 1989 [11]), and 8-22 months post-injury (Wilson et al., 1991 [14]). Two studies were not specifically related to time elapsed since injury: < 22 days-63 days (Pierce et al., 1990 [10]) and "at least 72 hours after injury" (Sisson, 1990 [12]). Though no specific time frame has been defined by neuro-plasticity theorists, the study by Mitchell et al. (1990 [9]) was the only one in which the whole subject group received coma stimulation intervention within two weeks of injury (Mitchell, 1990 [9]). Interestingly, it was also the study in which the results were the most unambiguous and positive.

(20) The relationship between time post-injury of coma stimulation and its relationship to outcomes has been studied only secondarily in the literature cited above. However, one area of research that is closely related provides more direct analysis in this area. Comprehensive inpatient rehabilitation programs (involving physical, occupational, and speech therapies) have been studied comparing time of initiation of therapy to patient outcomes.

(21) In a landmark study by Cope and Hall (1982 [2]), results showed that total hospital days for patients admitted late (over 35 days post-injury) to an acute rehabilitation program $(n=20)$ were over twice as long as those patients admitted within 35 days to the same program $(n=16)$ (Cope \& Hall, 1982 [2]). In an additional study, Mackay et al. (1992 [8]) compared subjects who entered a formal, early rehabilitation program $(n=17)$ to subjects who had not entered such a program $(n=21)$. These researchers found that patients in the formalized program had comas and rehabilitation stays approximately one-third the length of those in non-formalized programs, mean cognitive levels at discharge from acute hospitals and rehabilitation facilities that were significantly higher, and a significantly higher percentage of discharges to the home versus an extended care facility (Mackay et al., 1992 [8]). Though it is not the purpose of this paper to extensively review the literature related to comprehensive rehabilitation outcomes, the results in relationship to time elapsed post-injury before beginning intervention possibly triangulate to the field of coma stimulation.

\section{Annotated Critical References}

(22) Cope, D.N. \& Hall, K. (1982 [2]). Head injury rehabilitation: Benefit of early intervention. Archives of Physical Medicine and Rehabilitation, 63(9), 433-437.

(23) In this retrospective study, subjects were divided into early and late rehabilitation admission groups (admitted before or after 35 days post-injury). The early group consisted of 16 patients, and the late group consisted of 20 patients. Subjects were matched for age, level of disability, coma, neurosurgical procedures required, and other factors that might suggest less morbidity in the early admission group. Results showed that late admission patients required acute rehabilitation length of stay that was twice that of the early admission group. Both groups remained comparable in outcome two years post-injury. (Outcome was measured using formal tools such as the Disability Rating Scale and Glasgow Outcome Scale, as well as through functional parameters such as continence and social status outcome.) The authors speculated that early rehabilitation admission could result in substantial cost savings to the health care system as a whole.

(24) Kater, K.M. (1989 [7]). Response of head-injured patients to sensory stimulation. Western Journal of Nursing Research, 11 (1), 20-33.

(25) Kater conducted an experimental study $(n=15)$ using multimodal stimulation. The experimental group was matched on a one-to-one basis with the patients in the control group on the basis of sex, age range, approximate type of injury, Glasgow Coma Scale Score, and length of time post-injury. In addition to the treatment main effect and coma severity level main effects described above, Kater conducted an Experience and Interest Inventory history with a family member of each patient. This questionnaire included school subjects, activities, and 
amusements the patient experienced prior to head injury. Patients who experienced $50 \%$ or more of the items listed in the questionnaire were defined as coming from enriched environments. Kater found that the control group patients from enriched environments had significantly higher cognitive function scores after three months than those who were not from enriched environments.

(26) Mitchell, S., Bradley, V.A., Welch, J.L., \& Britton, P.G. (1990 [9]). Coma arousal procedure: A therapeutic intervention in the treatment of head injury. Brain Injury, 4 (3), 273-279.

(27) These researchers conducted an experimental study of multimodal, sensory stimulation sessions lasting one to two hours daily. Subjects in two groups $(n=12$ for each) were closely equated in terms of age, sex, type and location of head injury, surgical intervention, and Glasgow Coma Scale Score on admission. Relatives provided the stimulation using coma kits containing simple devices such as wooden blocks, warm and cold packs, and spices, for example. Results indicated that total duration of coma was significantly shorter, and that coma lightened more rapidly for the experimental group. The researchers hypothesized that coma arousal procedure could also have an effective role in reducing levels of distress for family members of the injured patient.

(28) Pierce, J.P., Lyle, D.M., Quine, S., Evans, N.J., Morris, J., \& Fearnside, M.R. (1990 [10]). The effectiveness of coma arousal intervention. Brain Injury, 4 (2), 191-197.

(29) This quasi-experimental study compared outcomes for 31 coma patients who were entered into a coma arousal program with a historical reference group of 135 similarly classified patients. The two outcomes measured were patient's Glasgow Outcome Scale Score 10-12 months post-injury and time taken to obey a simple command on two consecutive occasions 24 hours apart. Differences between the pilot study and reference group patients on initial characteristics suggested that the pilot study patients might have the more favorable outcomes independent of treatment effect. Stimulation was multimodal, delivered by family members, and lasted up to eight hours per day for seven days a week. Emphasis was placed on including stimuli with which the patient had previously been familiar. Findings indicated that no significant improvements were noted in either the time to obey a simple command or in the Glasgow Outcome Scale.

(30) Rader, M.A., Alston, J.B., \& Ellis, D.W. (1989 [11]). Sensory stimulation of severely brain-injured patients. Brain Injury, 3 (2), 141-147.

(31) The research reported in this article consisted of three separate studies. A first pilot study $(n=6)$ found that immediate changes in responsiveness occur as a result of sensory stimulation and that variables such as patient's body position and level of contact with the person providing stimulation have an effect on responsiveness. Supine position with high interpersonal contact was associated with the best changes in response level. The second study established validity and reliability values for the procedures. The third study measured General Responsiveness for 19 patients and then remeasured these same patients after three months. No significant differences were found in mean
General Responsiveness values. Relationships were reviewed between General Responsiveness and amount of treatment per day, frequency of family visits, pre-morbid education, age, time since injury, and neurological status. No differences were found between those who improved and those who did not.

\section{Practice Implications}

(32) Given the role that coma stimulation may have in rehabilitation outcomes, stimulation interventions should be considered when implementing a treatment plan. How might coma stimulation be practically implemented given the research described above?

(33) Firstly, multimodal stimulation should be the choice over unimodal stimulation when feasible with a patient. Both multimodal and unimodal stimulation have received attention in the literature. However, the study designs using multimodal stimulation are stronger than those using unimodal stimulation and have more unequivocal results. In addition, one researcher studied both multimodal and unimodal stimulation with the same subjects and found multimodal to increase responsiveness while unimodal stimulation had no effect (Wilson et al., 1991 [14]).

(34) Though the research reviewed gave several examples of multimodal stimulation, there is not research that has determined which of many individual options may lead to the highest increase in patient responsiveness. In implementing a multimodal stimulation program for a patient, then, nurses have an opportunity to use their creativity in stimulating each sense. Visual stimulation could be administered with a flashlight to the eyes or holding up brightly colored objects, a mirror, or large pictures with easily distinguishable shapes and objects. The patient can be encouraged to track these things as well. Auditory stimulation can be provided with taped voices of family and friends, favorite music, nature sounds, a tuning fork, or simple instruments such as wooden blocks. Olfactory stimulation could be perfume, spices, and foods. These same swabs of appropriate items could then be touched on the patient's tongue to stimulate the taste sensation. (It should be recalled that Wilson et al. 1991 [14] noted hypersensitivity when stimulating the taste sense, and care should be taken when stimulating for this reason.) Tactile stimulation could be administered by rubbing different materials, such as fur, felt, satin, or smooth metal (like the back of a spoon) as well as cool and warm temperatures over various patient body surfaces: arms, cheeks, back, etc.

(35) Because there are currently no guidelines in the literature regarding time spent in stimulation, nurses should take care that the patient has adequate blocks of rest and does not appear overly fatigued. However, it should also be remembered that some of the positive outcomes of these studies (eye opening and spontaneous motor activity, for example) are sometimes seen by nurses as signs of patient agitation. It is important to remember that increased activity and responsiveness are the goal of a coma stimulation program, and not continuous calm rest.

(36) Coma stimulation can also be incorporated into a nurse's daily routine (Davis \& White, 1995 [4]; Sosnowski \& Ustik, 1994 [13]). This sort of coma stimulation program would be especially helpful on units in which time spent with each 


\section{Table 1: Research Addressing Coma Stimulation}

\begin{tabular}{|c|c|c|c|c|c|}
\hline Authors & Design & Subjects & Time post-injury & Stimulation type & Outcomes \\
\hline $\begin{array}{l}\text { Mitchell } \\
\text { et al. } \\
(1990)[9]\end{array}$ & Experimental & $\mathrm{n}=12$ & 4-12 days & $\begin{array}{l}\text { Multimodal } 1-2 \mathrm{hr} \text { qd; } \\
\text { family administered }\end{array}$ & $\begin{array}{l}\text { Decrease mean coma } \\
\text { length, faster increase } \\
\text { in Glasgow scores in } \\
\text { experimental group }\end{array}$ \\
\hline $\begin{array}{l}\text { Kater } \\
(1989)[7]\end{array}$ & Experimental & $\mathrm{n}=15$ & at least two weeks & $\begin{array}{l}\text { Multimodal } 45 \text { min } \\
\text { twice qd for } 6 \text { days } \\
\text { x } 1-3 \text { months; staff } \\
\text { and family administered }\end{array}$ & $\begin{array}{l}\text { Increase in cognitive } \\
\text { level score in } \\
\text { experimental group }\end{array}$ \\
\hline $\begin{array}{l}\text { Rader et al. } \\
\text { (1989) [11] }\end{array}$ & $\begin{array}{l}\text { Quasi- } \\
\text { experimental }\end{array}$ & $\mathrm{n}=19$ & 2-33 months & $\begin{array}{l}\text { Multimodal } 50 \text { min qd } \\
\text { x } 2 \text { days; researcher } \\
\text { administered }\end{array}$ & $\begin{array}{l}\text { No improvement in } \\
\text { mean general } \\
\text { responsiveness }\end{array}$ \\
\hline $\begin{array}{l}\text { Pierce et al. } \\
\text { (1990) [10] }\end{array}$ & $\begin{array}{l}\text { Quasi- } \\
\text { experimental }\end{array}$ & $\mathrm{n}=31$ & $<22$ days-63 days & $\begin{array}{l}\text { Multimodal 3-8 hr qd } \\
\text { x 2-32 weeks; family } \\
\text { administered }\end{array}$ & $\begin{array}{l}\text { No significant difference } \\
\text { between groups } \mathrm{R} / \mathrm{T} \\
\text { recovery or time to } \\
\text { emerge from coma }\end{array}$ \\
\hline \multirow[t]{2}{*}{$\begin{array}{l}\text { Wilson et al. } \\
\text { (1991) [14] }\end{array}$} & $\begin{array}{l}\text { Quasi- } \\
\text { experimental }\end{array}$ & $\mathrm{n}=4$ & 8-22 months & $\begin{array}{l}\text { Multimodal } 10 \text { min qd } \\
\text { x } 11 \text { days; researcher } \\
\text { adminsitered }\end{array}$ & $\begin{array}{l}\text { Increase in } 1 \text { or more } \\
\text { of } 5 \text { behavioral } \\
\text { measures }\end{array}$ \\
\hline & & & & $\begin{array}{l}\text { Unimodal } 10 \text { min } \\
\text { qd x } 11 \text { days; } \\
\text { researcher administered }\end{array}$ & $\begin{array}{l}\text { No increase in } \\
\text { behavioral measures }\end{array}$ \\
\hline $\begin{array}{l}\text { Sisson } \\
\text { (1990) [12] }\end{array}$ & $\begin{array}{l}\text { Quasi- } \\
\text { experimental }\end{array}$ & $\mathrm{n}=5$ & at least 72 hours & $\begin{array}{l}\text { Unimodal } 3 \text { min qd } \\
\text { x } 5 \text { days; researcher } \\
\text { administered }\end{array}$ & $\begin{array}{l}\text { Either a change in EEG } \\
\text { or behavioral measure } \\
\text { with each subject }\end{array}$ \\
\hline $\begin{array}{l}\text { Jones et al. } \\
\text { (1994) [6] }\end{array}$ & $\begin{array}{l}\text { Quasi- } \\
\text { experimental }\end{array}$ & $\mathrm{n}=1$ & 42 days & $\begin{array}{l}\text { Unimodal } 5 \text { min } \\
\text { twice qd x } 28 \text { days; } \\
\text { researcher } \\
\text { administered }\end{array}$ & $\begin{array}{l}\text { Increase in arousal } \\
\text { following familiar } \\
\text { voices }\end{array}$ \\
\hline
\end{tabular}

patient is limited. For instance, a typical morning routine would provide mouth care (gustatory), a bath (tactile), a breakfast tray for a roommate (olfactory), a visit from family members, or a friendly monologue by the nurse (auditory and visual). A creative nurse could avoid duplication of stimulation from day to day with a modicum of time and planning.

(37) Secondly, stimulation programs should involve persons, obects, and other sensory stimuli that are familiar to the patient to the extent that is possible. Perception of a stimulus is based on recognizing its relationship to remembered personal experiences (Kater, 1989 [7]), and two studies used family and friends administering stimulation with positive results (Kater, 1989 [7]; Mitchell et al., 1990 [9]). An additional study using family members to administer the stimulation (Pierce et al., 1990 [10]) recorded small positive results that did not reach statistical significance. In addition, there may be some as yet unstudied benefit to patients' families and friends being involved in a coma stimulation program, perhaps alleviating feelings of distress, loss, anger, denial, and guilt, as suggested by Mitchell et al. (1990[9]).

(38) Finally, a coma stimulation program should be initiated as soon as the patient is medically stable. Though the coma stimulation research does not address this question directly, there is theoretical basis and rehabilitation research that supports early intervention (Cope \& Hall, 1982 [2]; Mackay et al., 1992 [8]). In addition, the experimental study by Mitchell et al. (1990 [9]) administered coma stimulation to patients the soonest (within 412 days following injury) with positive results. Ensuring that the patient is medically stable, initiating stimulation during the acute hospital stay may result in increased functional abilities, while waiting for admission to a rehabilitation program may result in a missed window of opportunity that cannot be regained. 


\section{Research Needed}

(39) Despite the gains that have been made in the area of coma stimulation, there are several factors that require further study. Firstly, a standardization of outcome measures would simplify cross comparison of studies and increase the likelihood of implementation. A standardization of measures might also increase the likelihood of larger sample sizes, which are sorely lacking. In addition, more focus related to functional outcome measures (such as ability to perform ADLs, hold a meaningful conversation, etc.) would increase both implementation and familial participation.

(40) Secondly, questions remain related to time frames. A clear relationship between time elapsed since injury and outcome following coma stimulation would prove invaluable in determining patients most likely to benefit from a coma stimulation program. Additionally, research should focus on the length of each stimulation session to provide maximum benefit, yet prevent patient fatigue or medical complications.

(41) Thirdly, research can be done on the stimulation itself, as well. For instance, could a video tape of a family member provide the same beneficial stimulation as the actual presence of the family member? Would bringing the patients outdoors in fine weather provide tactile stimulation equivalent or superior to stroking their extremities with various textured items?

(42) Finally, as partly alluded to by Mitchell et al. (1990 [9]), the impact of coma stimulation on persons other than the patient needs to be studied. The involvement of family members in a coma stimulation program may alleviate the family's feelings of distress, loss, guilt, denial, and anger (Mitchell et al., 1990 [9]). Additional parties that could be affected by coma stimulation programs are nursing staff (with regard to job satisfaction and staffing patterns, for example) and insurance providers, who are increasingly concerned with measurable, positive outcomes.

\section{Search Strategies}

\section{Search Terms:}

(43) coma, stimulation, brain injuries, head injuries, arousal, plasticity

\section{Databases Used:}

(44) MEDLINE (1985-96), English; CINAHL (1985-96), English

\section{References}

1. Ansell, B.J. (1991). Slow-to-recover brain injured patients: Rationale for treatment. Journal of Speech and Hearing Research, 34(5), 1017-1022. [MEDLINE Reference]

2. Cope, D.N. \& Hall, K. (1982). Head injury rehabilitation: Benefit of early intervention. Archives of Physical Medicine and Rehabilitation, 63(9), 433-437. [MEDLINE Reference]
3. Cotman, C.W. \& Nieto-Sampedro, M. (1982). Brain function, synapse renewal, and plasticity. Annual Review of Psychology, 33, 371-401. [MEDLINE Reference]

4. Davis, A.E. \& White, J.J. (1995). Innovative sensory input for the comatose brain-injured patient. Critical Care Nursing Clinics of North America, 7 (2), 351-361. [MEDLINE Reference]

5. Jennett, B. \& Plum, F. (1972). Persistent vegetative state after brain damage. Lancet, 1(753), 734-737.

[MEDLINE Reference]

6. Jones, R., Hux, K., Morton-Anderson, K.A., \& Knepper, L. (1994). Auditory stimulation effect on a comatose survivor of traumatic brain injury. Archives of Physical Medicine and Rehabilitation, 75(2), 164-171.

[MEDLINE Reference]

7. Kater, K.M. (1989). Response of head-injured patients to sensory stimulation. Western Journal of Nursing Research, 11 (1), 20-33. [MEDLINE Reference]

8. Mackay, L.E., Bernstein, B.A., Chapman, P.E., Morgan, A.S., \& Milazzo, L.S. (1992). Early intervention in severe head injury: Long-term benefits of a formalized program. Archives of Physical Medicine and Rehabilitation, 73(7), 635-641. [MEDLINE Reference]

9. Mitchell, S., Bradley, V.A., Welch, J.L., \& Britton, P.G. (1990). Coma arousal procedure: A therapeutic intervention in the treatment of head injury. Brain Injury, 4 (3), 273279. [MEDLINE Reference]

10. Pierce, J.P., Lyle, D.M., Quine, S., Evans, N.J., Morris, J., \& Fearnside, M.R. (1990). The effectiveness of coma arousal intervention. Brain Injury, 4 (2),191-197.

[MEDLINE Reference]

11. Rader, M.A., Alston, J.B., \& Ellis, D.W. (1989). Sensory stimulation of severely brain-injured patients. Brain Injury, 3 (2), 141-147. [MEDLINE Reference]

12. Sisson, R. (1990). Effects of auditory stimuli on comatose patients with head injury. Heart and Lung, 19 (4), 373-378. [MEDLINE Reference]

13. Sosnowski, C. \& Ustik, M. (1994). Early intervention: Coma stimulation in the intensive care unit. Journal of Neuroscience Nursing, 26 (6), 336-341. [MEDLINE Reference]

14. Wilson, S.L., Powell, G.E., Elliott, K., \& Thwaites, H. (1991). Sensory stimulation in prolonged coma: Four single case studies. Brain Injury, 5 (4), 393-400. [MEDLINE Reference]

\section{AUTHOR'S INFORMATION}

Sara Bos, MS, BSN, is an Adjunct Lecturer at the University of Michigan, School of Nursing, 2423 Stone, Ann Arbor, MI 48105. Fax: 313-763-1368. Email: sbos@umich.edu 